\title{
Affinage et qualité du gruyère de Comté VI. Caractéristiques sensorielles des fromages
}

\author{
J.L. BERDAGUE * et R. GRAPPIN \\ avec la collaboration technique de G. DUBOZ \\ INRA, Station Expérimentale laitière, 39800 Poligny, France \\ " Institut Technique du Gruyère
}

\section{Résumé}

L'étude porte sur 96 fromages affinés, représentant 24 fabrications, réalisées dans 4 fromageries, sur une période de 6 mois. A chaque fabrication, 4 fromages obtenus à partir d'une même cuve ont été affinés par 4 affineurs différents. Les fromages ont été notés par un jury de 15 personnes sur les critères sensoriels suivants : fruité, piquant, amer, mauvais goût et fermeté de la pâte en utilisant une échelle structurée à 5 barreaux.

Les analyses sensorielles ont été réalisées par comparaison directe des fromages provenant de fromageries différentes avec affinage identique et par comparaison directe de fromages provenant d'une même fromagerie ayant subi des affinages différents. Ces deux protocoles de dégustation ont donné des résultats identiques.

Malgré le fait que les fromages aient été affinés dans des conditions très différentes, ce facteur apparaît moins important, pour la plupart des caractéristiques sensorielles que le facteur fromagerie. Les caractères fruité et mauvais goût sont les seuls critères significativement influencés par les conditions d'affinage.

L'analyse de 48 fromages fabriqués en hiver montre l'existence d'une corrélation positive entre le caractère fruité de la pâte et la teneur en azote soluble dans l'acide phosphotungstique $(\mathrm{r}=0,56)$.

Pour 24 fromages fabriqués dans une fromagerie qui a développé une intense amertume durant la période estivale, l'amertume est apparue corrélée positivement avec $\log _{10}$ de la teneur en azote soluble dans l'eau $(r=0,72)$ et avec le pourcentage de caséines $\gamma 1+\gamma 2(\mathrm{r}=0,66)$.

Mots clés: Fromage - Gruyère de Comté - Analyses sensorielles - Protéolyse.

\section{Summary}

Ripening and quality of Gruyère de Comté cheese

VI. Sensory evaluation

96 mature cheeses, representing 24 batches of 4 cheeses were produced during a 6 month-period by 4 cheese plants and ripened in 4 different ripening operations.

Ce programme a reçu le soutien financier de la Direction des Industries Agricoles et Alimentaires du ministère de l'Agriculture (Contrat DIAA $\mathrm{n}^{\circ}$ 81/27) et de l'Association Nationale pour la Recherche et la Technologie (Bourse CIFRE de J.L. Berdague, Convention $n^{\circ}$ 92/82). 
The cheeses were tested by a panel of 15 persons for: Comté flavour intensity, piquant, bitterness, off-flavour and firmness, using for each criterium a five-point category rating scale.

For each trial sensory tests were carried out by direct comparisons of cheeses made in the different cheese plant and ripened in the same conditions. It was demonstrated that identical results can be obtained when direct comparisons of the ripening conditions instead of cheese plants were carried out.

Despite the fact that the cheeses were ripened in rather different conditions, this factor was less important for most of the sensory characteristics, than the origin of the cheese. Only, Comté flavour intensity and off-flavour were significantly influenced by the conditions of the ripening.

Based upon the analysis of 48 cheeses made in winter, Comté flavour intensity was positively correlated with the soluble phosphotungstic acid (PTA) nitrogen content of cheese $(\mathrm{r}=0.56)$.

For 24 cheeses made by one cheese plant which developped intense bitterness during the summer period, bitterness intensity was found positively correlated with $\log _{10}$ water soluble nitrogen content $(r=0.72)$ and with the proportion of $\gamma 1+\gamma 2$ caseins $(r=0.66)$.

Key words: Cheese - Gruyère of Comté - Sensory analysis - Proteolysis.

\section{Introduction}

Cet article qui s'insère dans le cadre des travaux sur l'affinage et la qualité du fromage de Comté (Grappin et al., 1987 ; Berdague et Grappin, 1987 ; Berdague et al., 1987 ; Collin et al., 1987; Guichard et al., 1987) a pour objectif :

- d'établir ou de confirmer des liens existants entre les caractéristiques physico-chimiques et la qualité organoleptique des fromages, appréciée par un jury de dégustateurs ;

- de préciser l'influence des facteurs fromagerie (qui intègre les caractéristiques de l'atelier et la technologie de fabrication), saison de fabrication et conditions d'affinage, sur certaines caractéristiques sensorielles du Gruyère de Comté.

\section{Matériel et méthodes}

Le protocole expérimental de fabrication des fromages a été décrit en détail par Grappin et al. (1987). Rappelons que les 96 fromages de l'étude représentent 24 fabrications réalisées dans 4 fromageries (F1, F2, F3 et F4) sur une période de 6 mois ( 3 mois d'hiver et 3 mois d'été). Au cours de chaque fabrication, 4 fromages ont été obtenus à partir d'une même cuve. Ces 4 fromages, considérés comme étant de composition identique, ont été affinés par 4 affineurs différents (A1, A2, A3 et A4). Le schéma expérimental correspond à un plan factoriel dont les niveaux sont répartis comme suit : Fromagerie $(4$ niveaux $) \times$ Affinage $(4$ niveaux $) \times$ Saison de fabrication $(2$ niveaux $) \times$ mois (3 répétitions). 


\section{A. Protocole des analyses sensorielles}

Les fromages ont été dégustés par groupes de quatre afin de ne pas créer une contrainte trop forte sur le jury. Comme la durée d'affinage variait avec le type d'affinage (4 à 10 mois), les comparaisons gustatives ont porté directement sur les fromages fabriqués par les 4 fromageries le jour $\mathbf{J}$ et affinés par le même affineur.

En conséquence, la comparaison entre les types d'affinage sera une comparaison indirecte inter-dégustation.

Pour vérifier la validité des conclusions qui seront portées sur l'influence de l'affinage à partir d'analyses sensorielles basées sur des comparaisons directes entre fromageries, une série de 48 fromages correspondant aux fabrications du jour $\mathbf{J}+1$ des mois d'été a été stockée au froid. Le stockage a permis de conserver les fromages à affinage rapide en attendant que s'achève celui des fromages à affinage long. Exception faite de l'effet du stockage à $6^{\circ} \mathrm{C}$ en cave froide, il est alors possible de faire une comparaison directe de l'influence du type d'affinage sur les qualités organoleptiques des fromages.

\section{B. Caractéristiques sensorielles des fromages}

L'ensemble des fromages de l'expérimentation constitue un bloc équilibré sur lequel ont porté les analyses. Les évaluations sensorielles étaient de type analytique descriptives, utilisant une échelle de notation à 5 barreaux (intervalles structurés), dans une perspective d'analyse qualitative-quantitative, les notes variant de 0 à 4 . Le jury était formé d'un groupe de 15 personnes expérimentées ( 6 femmes et 9 hommes, ayant entre 24 et 62 ans), appartenant à la profession fromagère ou au laboratoire INRA de Poligny. Chaque juge était choisi parmi 21 personnes ayant subi trois séances d'entraînement de 30 minutes environ. Ces séances ont comporté des épreuves de différenciation entre solutions amères et acides, ainsi que trois épreuves durant lesquelles des fromages très différents ont été dégustés afin de définir au maximum cinq caractéristiques sensorielles perçues de manière nette par l'ensemble du jury et présentant un intérêt commercial.

Les caractéristiques sensorielles sur lesquelles ont porté les notations sont les suivantes :

- piquant : sensation perçue sur la langue pendant la mastication ;

- amer: sélection des juges au seuil G4 (AFNOR V09-002/1980) de dilutions de chlorhydrate de quinine. Des solutions de naringine (FLUKA: qualité pure), principe amer du pamplemousse, ont également été utilisées aux concentrations de $0,036,0,018$ et $0,009 \mathrm{~g} / \mathrm{l}$. Afin d'éviter la confusion fréquente entre les termes acide et amer, une sélection des juges au seuil G4 (AFNOR V09-002/1980) de dilution d'acide tartrique et d'acide citrique a été réalisée ;

- fruité : ce vocable souvent utilisé par les professionnels désigne la saveur typique du Comté et l'intensité générale de saveur agréable du fromage ; 
- mauvais goût : il s'agit d'un goût désagréable de nature diverse (petit lait, rance, savon, pomme de terre, etc.) et la plupart du temps non qualifiable.

Les dégustations ont eu lieu dans un local climatisé à $18^{\circ} \mathrm{C}$, les boxes de dégustation individuels étant éclairés en lumière jaune, afin d'éliminer une interaction possible entre la couleur des fromages et les autres caractéristiques sensorielles.

Les fromages étaient présentés dans des boîtes de Petri de $14 \mathrm{~cm}$ de diamètre, sous forme de 20 à 30 cubes de $1 \mathrm{~cm}$ d'arête. Pour obtenir des morceaux de qualité à peu près homogène, une zone sous-croûte de $2 \mathrm{~cm}$ était éliminée de la portion à déguster. Les fromages découpés en cube restaient une heure à température de la pièce avant chaque séance.

Entre chaque dégustation, il était recommandé aux juges de se rincer la bouche (eau de Volvic). Des tranches de pain étaient à disposition lors de la dégustation.

\section{Analyse statistique}

Chaque fromage s'est vu attribuer une note correspondant à la moyenne des notes du jury. Chaque moyenne est intégrée dans l'étude statistique au même titre qu'une valeur analytique. L'exploitation statistique fait intervenir les techniques décrites par GrapPIN et al. (1987).

\section{Résultats et discussion}

\section{A. Moyennes et variations}

Pour l'ensemble de l'expérimentation, les fromages ont obtenu une note de fruité égale à $2,6 / 4$, alors que les caractères piquant, amer ou mauvais goût ont des notes comprises entre $1,7 / 4$ et $1,3 / 4$. La pâte a été trouvée assez ferme : 2,7/4. Les caractères fruité et fermeté de la pâte ont la plus forte amplitude de variation (fig. 1).

\section{B. Etude des relations entre les caractéristiques sensorielles et physico-chimiques des fromages}

Le choix des critères répond à des habitudes de la profession fromagère. Il est ambigu au sens où des termes comme amer ou ferme ont une signification précise après entraînement des juges, tandis que l'appréciation du caractère fruité, piquant ou du mauvais goût d'une pâte est beaucoup plus difficile.

Dans ce chapitre, l'ensemble des relations entre la composition chimique et la qualité des fromages sera présenté et discuté, même si les coefficients de corrélations sont le plus souvent faibles, quoique statistiquement significatifs. 


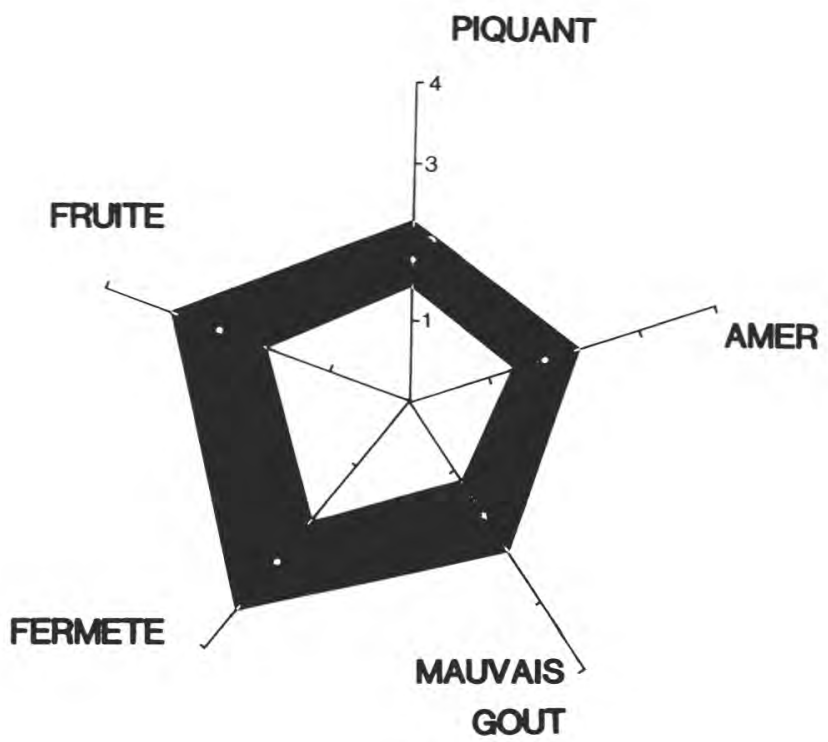

FIG. 1

Profil sensoriel des 96 fromages de Comté. La surface noire correspond à l'encadrement de la moyenne (rond blanc) plus ou moins un écart-type de la population.

Profile of sensory analysis of Comté Cheese. The black frame represents the average score (white spots) \pm 1 standard deviation for 96 cheeses.

Ces faibles corrélations proviennent d'une part de l'incertitude plus importante des résultats des analyses sensorielles par rapport aux analyses physico-chimiques et d'autre part des relations souvent non linéaires entre variables sensorielles et variables physico-chimiques.

\section{Les fractions azotées}

Les différentes fractions azotées mesurées dans cette étude (Collin et al., 1987) ont un rôle prépondérant sur la flaveur des fromages, en particulier au niveau des caractères fruité, piquant et amer (tabl. 1).

La fraction NPT (azote soluble dans l'acide phosphotungstique) qui représente essentiellement les acides aminés, est corrélée positivement au caractère fruité des fromages $(r=0,33)$. Cette faible valeur du coefficient de corrélation, calculée sur 96 fromages, est due à l'apparition pendant les mois d'été de saveur amère et de mauvais goût qui ont masqué en partie le caractère fruité. En effet, la corrélation entre le taux de NPT et le caractère fruité des fromages est plus élevée sur les 48 fromages fabriqués en hiver $(\mathrm{r}=0,56)$ que sur les 48 fromages d'été $(\mathrm{r}=0,32)$. Une telle relation positive entre la teneur en NPT et l'intensité de flaveur, a déjà été mentionnée par ASTON et al. (1983a,b) dans le cas du Cheddar. 
L'acide isovalérique, témoin de la dégradation de la leucine (BARAud et al., 1970) et donc d'une protéolyse avancée, est aussi corrélé positivement $(\mathrm{r}=0,27)$ au caractère fruité des fromages.

En ce qui concerne l'amertume, on note (tabl. 1) qu'elle est corrélée positivement au taux d'azote soluble, et aux fractions $\beta$ dégradées, $\gamma 2$ et $\gamma 3$. Si on ne considère que la population des 24 fromages de la fromagerie 4 qui a présenté ce défaut de façon nette en période estivale (fig. 4C), on obtient après transformation logarithmique des teneurs en azote soluble des fromages (Moskowitz et al., 1979; SAUvageot, 1982) une corrélation élevée $(r=0,72)$ avec l'amertume (fig. 2).

Sur cette même population, on obtient également un lien net (fig. 3) entre la somme des fractions $\gamma 2+\gamma 3$ et l'amertume des Comtés $(r=0,66)$.

\section{TABLEaU 1}

Relations entre les caractéristiques sensorielles et la composition physico-chimique des fromages en fin d'affinage $(n=96)$

Seuils de signification des coefficients de corrélation: ${ }^{*}=p<0,05 ;{ }^{* * *}=p<0,01$

Coefficient of correlation between sensory analyses and physico-chemical composition of Comté cheese $(n=96)$

* Significant $p<0.05 ;{ }^{* *}$ Significant $p<0.01$

\begin{tabular}{|c|c|c|c|c|c|c|}
\hline \multirow{2}{*}{\multicolumn{2}{|c|}{$\begin{array}{l}\text { Composition chimique } \\
\text { Chemical composition }\end{array}$}} & \multicolumn{5}{|c|}{$\begin{array}{l}\text { Caractéristiques sensorielles } \\
\text { Sensory characteristics }\end{array}$} \\
\hline & & \multirow{2}{*}{$\begin{array}{l}\begin{array}{c}\text { Fruité } \\
\text { Comté } \\
\text { flavour } \\
\text { intensity }\end{array} \\
0,11\end{array}$} & \multirow{2}{*}{$\begin{array}{l}\text { Piquant } \\
\text { Piquant } \\
-0,08\end{array}$} & \multirow{2}{*}{\begin{tabular}{|}
$\begin{array}{l}\text { Amer } \\
\text { Bitter }\end{array}$ \\
$0,28^{* *}$
\end{tabular}} & \multirow{2}{*}{\begin{tabular}{|c|}
$\begin{array}{c}\text { Mauvais } \\
\text { goût } \\
\text { Off-flavour }\end{array}$ \\
0,13 \\
\end{tabular}} & \multirow{2}{*}{$\begin{array}{l}\text { Fermeté } \\
\text { Firmness } \\
0,02\end{array}$} \\
\hline \multirow{7}{*}{ 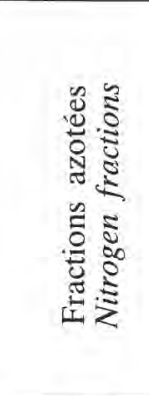 } & $\beta$ deg & & & & & \\
\hline & $\gamma 1$ & $-0,14$ & $-0,23^{*}$ & 0,01 & 0,08 & $-0,17$ \\
\hline & $\gamma 2$ & 0,18 & $-0,22^{*}$ & $0,31^{* *}$ & 0,19 & $-0,07$ \\
\hline & $\gamma 3$ & 0,25 & $-0,11$ & $0,21^{*}$ & 0,17 & $-0,12$ \\
\hline & $\alpha s_{1}-\mathrm{I}$ & $-0,19$ & 0,19 & $-0,09$ & 0,01 & $-0,12$ \\
\hline & NS & 0,08 & $-0,10$ & $0,48^{* *}$ & $0,22^{*}$ & $-0,15$ \\
\hline & NPT & $0,33^{* *}$ & $0,39 * *$ & 0,14 & 0,08 & 0,13 \\
\hline \multirow{5}{*}{ 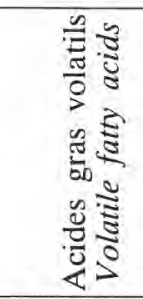 } & iC5 & $0,26^{* *}$ & $0,24^{*}$ & $-0,27^{* *}$ & 0,04 & $-0,07$ \\
\hline & $\mathrm{C} 2$ & $-0,19$ & $-0,00$ & $-0,03$ & $-0,22^{*}$ & 0,11 \\
\hline & $\mathrm{C} 3$ & $0,35^{* * *}$ & $-0,08$ & 0,02 & $-0,06$ & 0,13 \\
\hline & $\mathrm{C} 4$ & 0,19 & $-0,03$ & 0,11 & $-0,01$ & 0,04 \\
\hline & C6 & 0,17 & $-0,04$ & 0,12 & 0,01 & 0,03 \\
\hline $\begin{array}{l}\text { Sel } \\
\text { Salt }\end{array}$ & $\mathrm{Nacl}$ & $0,41^{* *}$ & $0,36^{* *}$ & $-0,35^{* *}$ & 0,11 & $0,27^{* *}$ \\
\hline
\end{tabular}




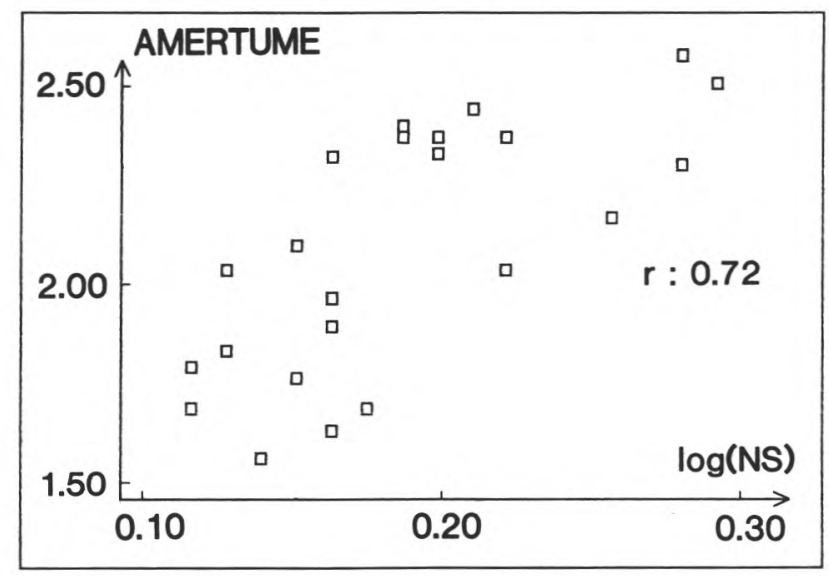

FIG. 2

Correlation entre l'amertume des fromages et le logarithme décimal des teneurs en azote soluble des 24 fromages de la fromagerie F4.

Relationship between bitterness and $\log _{10}$ water soluble nitrogen content of 24 cheeses from the cheese plant F4.

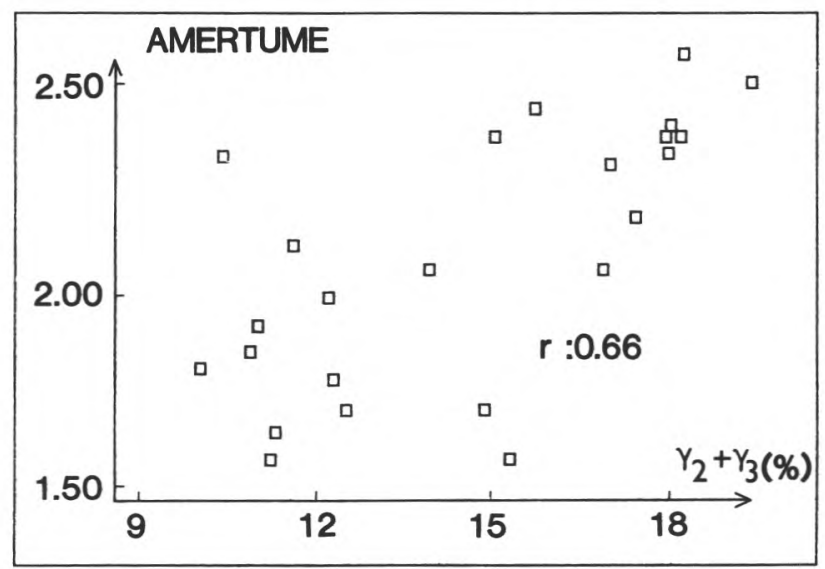

FIG. 3

Correlation entre l'amertume des fromages et la somme des pourcentages des caséines $\gamma 2$ et $\gamma 3$ des 24 fromages de la fromagerie $F 4$.

Relationship between bitterness and the proportion of $\gamma 2+\gamma 3$ caseins of 24 cheeses from cheese plant F4. 
Les fractions $\gamma 2$ et $\gamma 3$ correspondent respectivement aux séquences 106209 et 108-208 de la caséine provenant de l'hydrolyse de la caséine $\beta$ par la plasmine (Kaminogawa et YamauCHI, 1972a et b ; Eigel, 1977 ; Kaminogawa et al., 1980; GrapPIN et al., 1985). Etant donné la taille moléculaire des fractions $\gamma 2$ et $\gamma 3$, il est vraisemblable que celles-ci ne sont pas directement responsables de l'amertume. Ces fractions représentent plutôt une étape de la dégradation des caséines $\beta$ facilitant l'apparition de peptides amers dans les conditions physico-chimiques, microbiologiques et enzymatiques analogues à celles de la fromagerie F4. VisSER et al. (1983) signalent que la caséine $\beta$, et plus particulièrement sa région $C$ terminale est une source importante de peptides amers dans le fromage de Gouda. Les travaux de DrIESSEN (1983) sur lait UHT montrent qu'après stockage, la protéolyse due à la plasmine est responsable de l'apparition d'amertume des laits. Un temps de stérilisation court (faible dénaturation du plasminogène et de la plasmine), associé à une augmentation de la protéolyse, entraîne l'apparition de goûts amers dans le lait.

L'absence de traitement thermique du lait et un affinage long sont autant de conditions permettant une expression diversifiée des mécanismes protéolytiques, pouvant aussi conduire à l'apparition de peptides amers en Comté.

\section{Acides gras volatils}

Les teneurs en AGV (BERdague et al., 1987) n'apparaissent pas liées aux caractéristiques sensorielles (tabl. 1). Cela peut s'expliquer par le fait que les fromages de l'expérimentation ne présentaient pas d'anomalies fermentaires (fermentation propionique excessive engendrant une saveur piquante, ou fermentation butyrique caractérisée), ni une lipolyse anormale. La seule relation qui apparaît concerne le parallélisme entre un fort taux d'acide propionique et une note plus fruitée des Comtés $(r=0,35)$.

\section{Chlorure de sodium}

La teneur en sel a une incidence importante sur la qualité organoleptique des aliments. Son rôle bien connu d'agent de sapidité et d'exhausteur de goût semble se manifester aussi en Comté (tabl. 1). La teneur en sel apparaît corrélée positivement aux caractères fruité et piquant de la pâte, tandis qu'une corrélation négative est observée avec l'amertume des fromages.

Le rôle du sel est complexe. En effet, Noomen (1978) a montré sur fabrications expérimentales de fromages hollandais type Meshanger que, selon sa concentration, celui-ci agit soit comme activateur, soit comme inhibiteur de la protéolyse. L'action du sel sur le caractère amer des fromages, indépendamment de son rôle d'agent de sapidité qui peut masquer ce défaut, peut être plus profonde en orientant différemment certains mécanismes de protéolyse, induisant ou empêchant selon les cas, l'apparition d'amertume. Dans une étude sur les facteurs affectant l'amertume dans le fromage de Gouda, STAdhouders et Hup (1975) ont observé qu'une augmentation de la teneur en sel des fromages réduisait l'amertume produite par les levains lactiques. Si les mécanismes d'action du sel ne sont pas encore parfaitement expliqués, il a cependant été montré que l'hydrolyse des caséines $\beta$ par la présure est inhibée par le sel (Fox et Walley, 1971 ; Phelan et Guiney, 1973) et que la dégradation 
de la caséine $\beta$ par $S$. cremoris est aussi ralentie par le sel (Sullivan et Jago, 1972).

D'après Noomen (1978), l'action de la plasmine pourrait être considérablement affectée par les caractéristiques physico-chimiques des fromages, dont la teneur en sel. La fromagerie F4 qui a présenté le défaut d'amertume était aussi celle dont les teneurs en sel des fromages étaient les plus basses (Berdague et Grappin, 1987).

Le rôle de l'apport du sel durant l'affinage (mode d'apport, quantité et cinétique de pénétration dans la pâte), n'est pas abordé dans cette étude. L'existence de gradients de sel (Снамва, 1982) peut induire des gradients de protéolyse qui engendrent des gradients de saveur dans les fromages. De tels gradients peuvent contribuer à expliquer l'hétérogénéité organoleptique de la pâte, fréquemment signalée lors des dégustations.

\section{Influence des facteurs sur les caractéristiques sensorielles des fromages}

Les caractéristiques des différents niveaux des facteurs fromagerie, affinage et saison sont décrits dans l'article d'introduction de la série « Affinage et qualité du Gruyère de Comté »(GRAPPIN et al., 1987).

\section{TABLEAU 2}

Analyse de la variance des caractéristiques sensorielles des fromages

Influence of the factors, ripening conditions, cheese plant and season on the sensory characteristics of Comté cheese. Analysis of variance

\begin{tabular}{|c|c|c|c|c|c|c|}
\hline \multirow{3}{*}{$\begin{array}{l}\text { Variables } \\
\text { Variables }\end{array}$} & \multicolumn{6}{|c|}{$\begin{array}{l}\text { Facteurs } \\
\text { Factors }\end{array}$} \\
\hline & \multicolumn{2}{|c|}{$\begin{array}{l}\text { Affinage } \\
\text { Ripening }\end{array}$} & \multicolumn{2}{|c|}{$\begin{array}{l}\text { Fromagerie } \\
\text { Cheese Plant }\end{array}$} & \multicolumn{2}{|c|}{$\begin{array}{l}\text { Saison } \\
\text { Season }\end{array}$} \\
\hline & (1) & (2) & (1) & (2) & (1) & (2) \\
\hline $\begin{array}{l}\text { Fruité } \\
\text { Comté flavour intensity }\end{array}$ & $* *$ & 5,2 & $* *$ & 48,6 & $* *$ & 6,6 \\
\hline $\begin{array}{l}\text { Piquant } \\
\text { Piquant }\end{array}$ & NS & & $* *$ & 35,6 & NS & \\
\hline $\begin{array}{l}\text { Amer } \\
\text { Bitter }\end{array}$ & NS & & $* *$ & 32,4 & $* *$ & 6,4 \\
\hline $\begin{array}{l}\text { Mauvais goût } \\
\text { Off-flavour }\end{array}$ & $* *$ & 39,6 & $*$ & 8,7 & $* *$ & 7,3 \\
\hline $\begin{array}{l}\text { Fermeté } \\
\text { Firmness }\end{array}$ & NS & & $* *$ & 38,6 & NS & \\
\hline \multicolumn{7}{|c|}{$\begin{array}{l}\text { (1) Seuils de signification : non significatif (NS), significatif au seuil de } 5 \%\left({ }^{*}\right) \text { et } 1 \%\left({ }^{* *}\right) \text {. } \\
\text { (1) Significance levels: } *(5 \%) ; * \text {; }(1 \%) \text {. } \\
\text { (2) Pourcentage de la variance expliqué par chaque facteur. } \\
\text { (2) Percentage of the variance explained by each factor. }\end{array}$} \\
\hline
\end{tabular}


1. Evaluation sensorielle mettant en jeu une comparaison directe des fromageries

Cette comparaison comprend les 48 fromages d'hiver et les 48 fromages d'été, fabriqués le jour J. Les analyses de la variance, effectuées pour chaque variable (tabl. 2) montrent que la fromagerie est le facteur de variation dominant pour l'ensemble des caractéristiques sensorielles.

La figure 4 illustre l'effet fromagerie sur les caractères fruité (4a), piquant (4b), amer (4c) et fermenté (4d) des fromages. Les fromageries 2 et 4 se distinguent très nettement au niveau des goûts fruité et piquant. Il est intéressant de noter que les fromageries 2,3 et 4 donnent des produits dont le niveau de fruité ou de piquant perçu par les juges est assez stable au cours des mois, tandis que la fromagerie 1 présente une variabilité beaucoup plus importante. Au niveau de la texture de la pâte (fig. 4d), les fromageries 1 et 3 donnent en moyenne les fromages les plus fermes et on constate une variabilité importante de ce critère durant les mois d'hiver.

Les différences d'amertume observées sont dues uniquement à la fromagerie 4 qui a très nettement présenté ce défaut en été (fig. 4c).

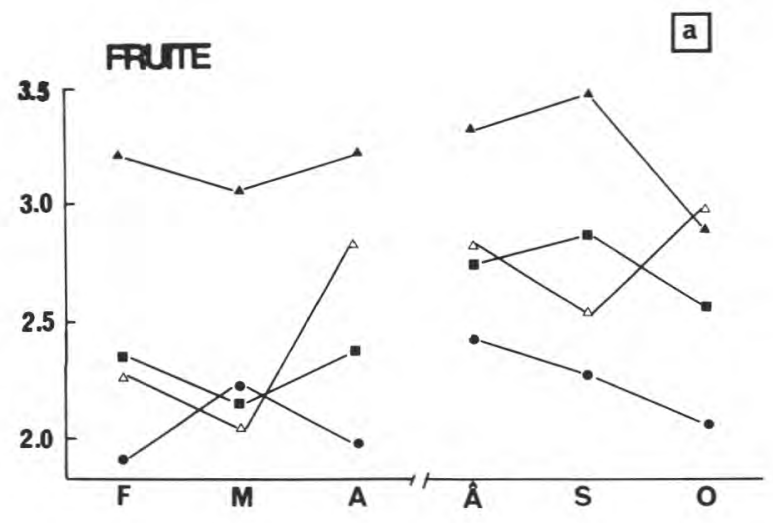

PIQUANT

b

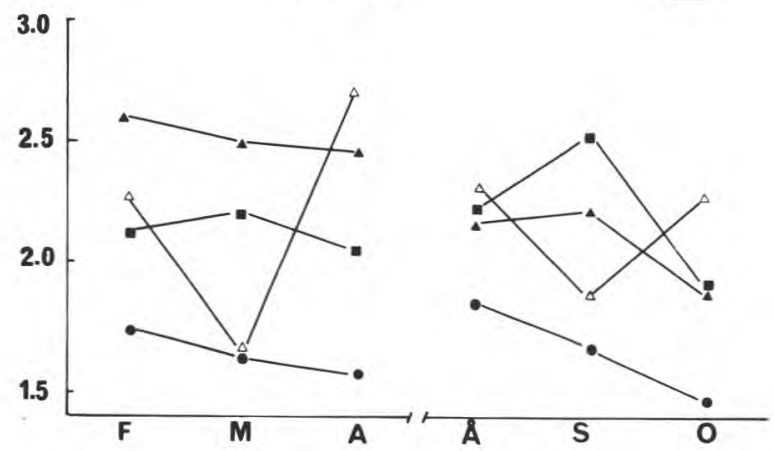


AMER

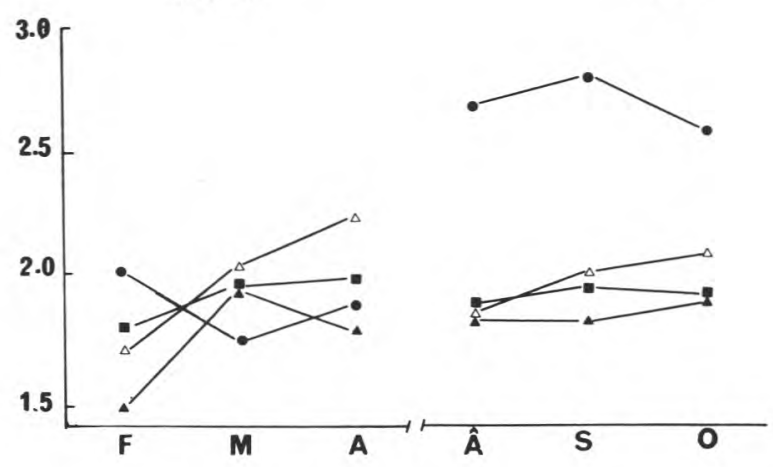

FEMETE

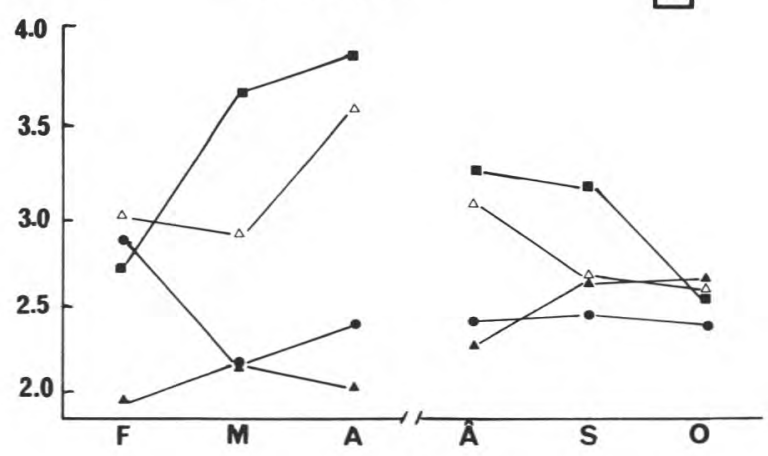

FIG. 4

Evolution moyenne mensuelle des caractéristiques sensorielles: fruité (a), piquant (b), amertume $(c)$ et fermeté $(d)$ des 96 fromages fabriqués dans les 4 fromageries. $F 1(\triangle), F 2(\mathbf{\Delta}), F 3(\square)$ et $F 4(\bullet)$.

Average monthly variation of sensory characteristics: Comté flavour intensity (a), piquant (b), bitterness $(c)$, firmness $(d)$, of the 96 cheeses, made in the 4 cheese plants, $F 1(\triangle), F 2(\mathbf{\Delta}), F 3(\mathbf{\square})$ and $F 4(\bullet)$.

L'effet saison est net sur le caractère fruité des fromages, particulièrement si l'on fait abstraction de l'affinage (fig. 5a). Les fromages fabriqués en été apparaissent plus fruités que les fromages d'hiver.

L'apparition de mauvais goût en période estivale est uniquement due à l'affineur 1 (fig. 5b).

L'effet affinage n'apparaît significatif que sur les caractères fruité et mauvais goût. Durant l'hiver, l'affinage 1 a conduit à des fromages plus fruités (fig. 5a). Cette différence par rapport aux autres types d'affinage n'existe plus 

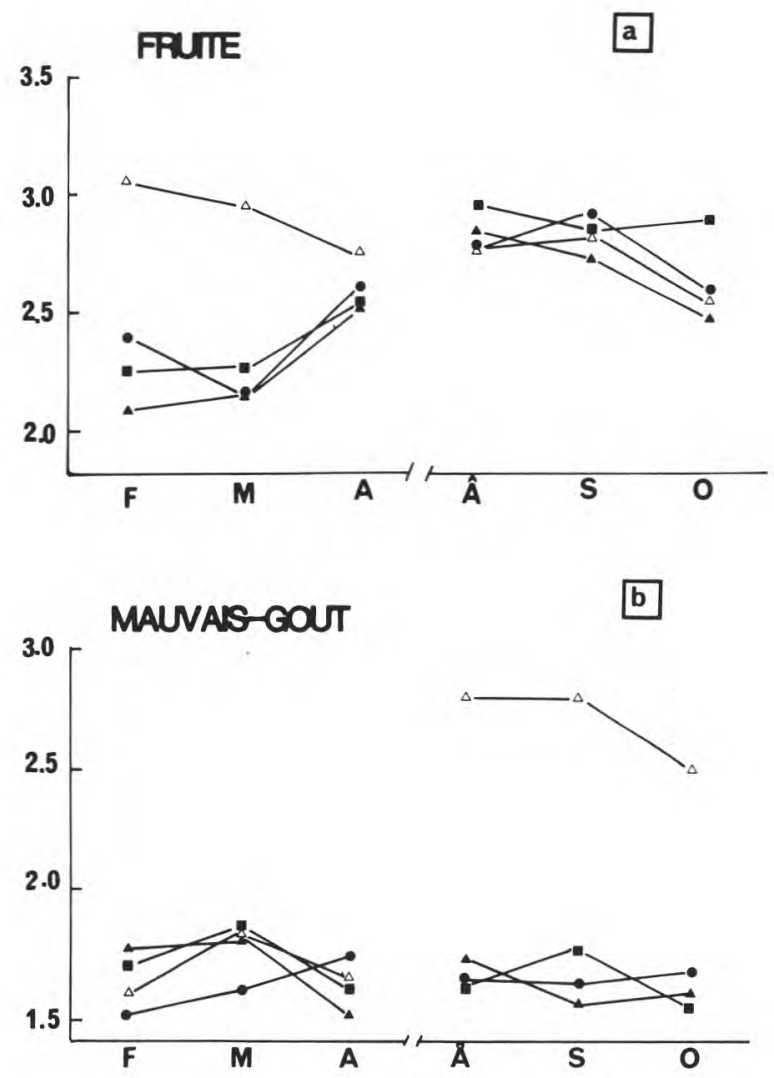

FIG. 5

Evolution moyenne mensuelle des caractéristiques sensorielles: fruité (a) et mauvais goût (b) des 96 fromages par type d'affinage, $A 1(\triangle), A 2(\mathbf{\Delta}), A 3(\mathbf{\square}), A 4(\mathbf{0})$.

Average monthly variation of sensory characteristics: Comté flavour intensity (a), and soft-flavour (b) of the 96 cheeses ripened in the 4 differents ripening operations, $A 1(\triangle)$, $A 2$ (山), $A 3$ (国), $A 4(\mathbf{0})$.

en été, en raison non seulement d'une amélioration de la qualité moyenne des fromages affinés par $\mathrm{A} 2, \mathrm{~A} 3$ et $\mathrm{A} 4$, mais vraisemblablement aussi à cause de l'apparition de mauvais goût chez A1 (fig. 5b). L'étude plus fine de la correlation positive entre le caractère fruité et la teneur en NPT des fromages fabriqués en hiver $(r=0,56)$, montre que la correlation observée (fig. 6) peut être due aussi bien aux particularités propres de la fromagerie F2, définies par la nature du lait et les conditions de fabrication (levains notamment), qu'aux conditions de l'affinage A1 (affinage long et température d'affinage basse).

Le mauvais goût n'a pas pu être relié aux composés volatils du Comté (Guichard et al., 1987) et ne présente pas de liens avec les autres analyses chimiques de l'étude (GrapPIN et al., 1987). 


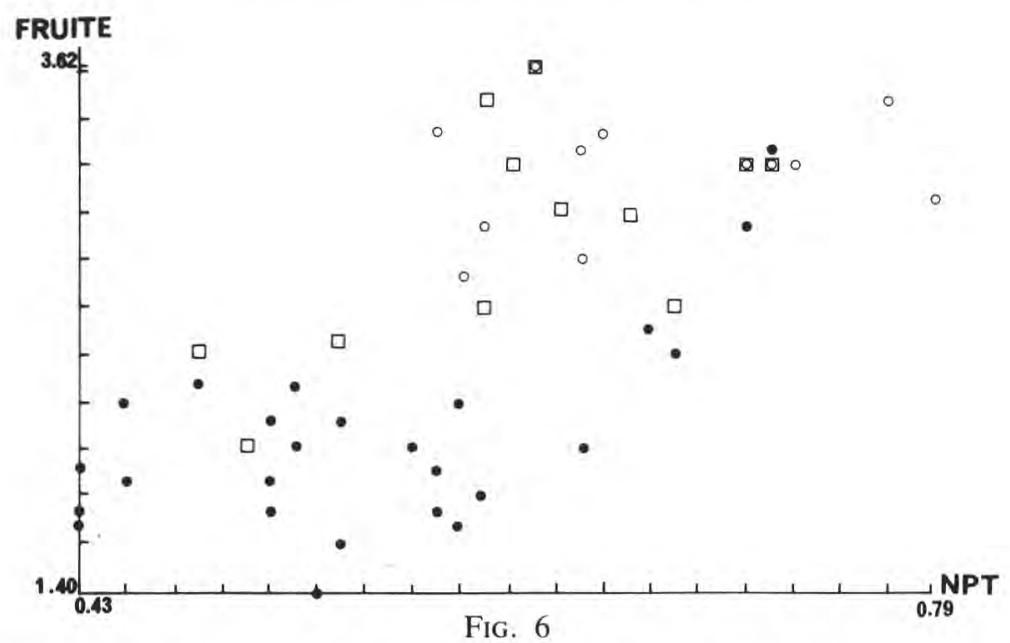

Correlation entre la note de fruité et la teneur en NPT des 48 fromages fabriqués en hiver.

(O) fromages fabriqués par F2, ( $\square$ ) fromages affinés par A1, (-) autres fromages.

Correlation between Comté flavour intensity score and PTA nitrogen content of 48 cheeses made during the winter period.

(O) cheeses made by F2, ( $\square$ ) cheeses ripened by A1, (०) others cheeses.

Les rôles respectifs de la fromagerie et du type d'affinage sont ici difficiles à évaluer puisque les dégustations permettent seulement une comparaison directe entre fromageries.

2. Evaluation sensorielle mettant en jeu une comparaison directe des conditions d'affinage

Si l'on compare les résultats des analyses de variance faites sur les analyses sensorielles réalisées, d'une part sur les fromages d'été non stockés (48 fromages, fabrication du jour J) et d'autre part sur les fromages d'été stockés ( 48 fromages fabrication du jour $\mathbf{J}+1$ ), on constate que les deux modes de dégustation (comparaison directe des fromageries et comparaison directe des affinages) conduisent à des résultats très proches (tabl. 3).

En faisant abstraction des modifications subies par les fromages durant les 2 mois et demi de stockage à basse température, on constate que des différences significatives existent selon le type d'affinage. Ces différences concernent les mêmes critères sensoriels : fruité, mauvais goût, qu'il s'agisse d'une comparaison directe ou indirecte du type d'affinage lors des dégustations. La comparaison directe entre types d'affinage a mis aussi en évidence des différences au niveau de la fermeté de la pâte selon les conditions d'affinage de la pâte. Il faut aussi souligner, sur le plan sensoriel, que le protocole expérimental (comparaison directe des fromageries ou comparaison directe des types d'affinage), ne perturbe pas le classement des moyennes (tabl. 3), et que d'une 


\section{TABLEAU 3}

Analyse de la variance: influence des facteurs fromagerie et affinage sur les $2 \times 48$ fromages d'été. Comparaison directe entre fromageries $(A)$ et comparaison directe entre types d'affinage $(B)$

Influence of ripening conditions, cheese plant and season on the sensory characteristics of $2 \times 48$ cheeses produced in summer. Analysis of variance (A) : Sensory evaluation made by comparing simultaneously cheeses from 4 different cheese plants, made at day $J$, and ripened in the same conditions.

(B) : Sensory evaluation made comparing simultaneously cheeses from the same vat made at day $J+1$, and ripened in different conditions.

\begin{tabular}{|c|c|c|c|c|c|c|c|c|c|c|c|c|}
\hline \multirow[b]{3}{*}{$\begin{array}{l}\text { Fruité } \\
\text { Comte flavour } \\
\text { intensity }\end{array}$} & \multirow[b]{3}{*}{$\begin{array}{l}(1) \\
(2)\end{array}$} & \multicolumn{5}{|c|}{$\begin{array}{c}\text { Comparaison directe } \\
\text { des fromageries } \\
\text { Direct comparison between } \\
\text { cheese plant } \\
\text { (A) }\end{array}$} & \multicolumn{6}{|c|}{$\begin{array}{c}\text { Comparaison directe } \\
\text { des types d'affinage } \\
\text { Direct comparison between } \\
\text { ripening types } \\
\text { (B) }\end{array}$} \\
\hline & & \multicolumn{2}{|c|}{$\begin{array}{l}\text { Affinage } \\
\text { Ripening }\end{array}$} & \multicolumn{3}{|c|}{$\begin{array}{l}\text { Fromagerie } \\
\text { Cheese plant }\end{array}$} & \multicolumn{3}{|c|}{$\begin{array}{l}\text { Affinage } \\
\text { Ripening }\end{array}$} & \multicolumn{3}{|c|}{$\begin{array}{l}\text { Fromagerie } \\
\text { Cheese plant }\end{array}$} \\
\hline & & $\stackrel{*}{*}$ & $\begin{array}{l}3,7 \\
3\end{array}$ & $\begin{array}{c}* * \\
4\end{array}$ & $\underline{13}$ & $\begin{array}{c}64,1 \\
2\end{array}$ & $\begin{array}{c}* * \\
1\end{array}$ & $\underline{24}$ & $\begin{array}{c}23,4 \\
3\end{array}$ & $\begin{array}{c}* * \\
4\end{array}$ & 1 & $\begin{array}{l}35,2 \\
\underline{32}\end{array}$ \\
\hline $\begin{array}{l}\text { Piquant } \\
\text { Piquant }\end{array}$ & $\begin{array}{l}(1) \\
(2)\end{array}$ & NS & - & $\begin{array}{l}* * \\
4\end{array}$ & & $\begin{array}{l}32,6 \\
123 \\
\end{array}$ & NS & & - & $\begin{array}{l}* \\
4\end{array}$ & & $\begin{array}{l}15,9 \\
123 \\
\end{array}$ \\
\hline $\begin{array}{l}\text { Amer } \\
\text { Bitter }\end{array}$ & $\begin{array}{l}(1) \\
(2)\end{array}$ & NS & - & $\begin{array}{l}* * \\
\underline{123} \\
\end{array}$ & & $\begin{array}{c}67,5 \\
4\end{array}$ & NS & & - & $\begin{array}{l}* * \\
123 \\
\end{array}$ & & $\begin{array}{c}66,1 \\
4\end{array}$ \\
\hline $\begin{array}{l}\text { Mauvais goût } \\
\text { Off-flavour }\end{array}$ & $\begin{array}{l}(1) \\
(2)\end{array}$ & $\begin{array}{c}* * \\
234 \\
\end{array}$ & $\begin{array}{c}64,3 \\
1\end{array}$ & NS & & - & $\begin{array}{c}* * \\
234 \\
\end{array}$ & & $\begin{array}{c}35,5 \\
1\end{array}$ & $\begin{array}{c}* \\
123 \\
\end{array}$ & & $\begin{array}{l}9,2 \\
4\end{array}$ \\
\hline $\begin{array}{l}\text { Fermeté } \\
\text { Firmness }\end{array}$ & $\begin{array}{l}(1) \\
(2)\end{array}$ & NS & - & $\begin{array}{l}* * \\
4\end{array}$ & & $\begin{array}{l}30,1 \\
123 \\
\end{array}$ & $\begin{array}{c}* * \\
124 \\
\end{array}$ & & $\begin{array}{c}16,5 \\
3\end{array}$ & $\begin{array}{c}* * \\
4\end{array}$ & $\underline{12}$ & $\begin{array}{c}27,9 \\
3\end{array}$ \\
\hline
\end{tabular}

(1) Seuil de signification : non significatif (NS), significatif au seuil de $5 \%\left({ }^{*}\right)$ et $1 \%\left(^{* *}\right.$ ), et pourcentage de variance expliqué par le facteur étudié.

(1) Significance levels $\left({ }^{*}: 5 \% ; * *: 1 \%\right)$ and percentage of the variance explained by each factor.

(2) Lorsque les différences entre moyennes sont significatives et en l'absence d'interactions significatives cntre les facteurs, une comparaison des moyennes a été effectuée (test de Newmann-Keuls). Les moyennes non significativement différentes sont soulignées.

(2) Ranking of the 4 types of ripening and 4 cheese plants. Underlined numbers indicate that the means are not significantly different at $5 \%$ probability (NEUWMANN-KEULS test).

façon générale, la fromagerie a une action prépondérante par rapport à l'affinage, comme en témoignent les pourcentages de variation expliqués par les différents facteurs (tabl. 3). Cependant, l'élimination de la partie corticale des fromages a probablement réduit l'influence des conditions d'affinage sur leurs caractéristiques organoleptiques, puisque près de la morge se trouve la plus grande concentration de composés aromatiques formés par la flore de surface. 


\section{Références bibliographiques}

Aston J.W., Durward I.G,, Dulley J.R., 1983a, Proteolysis and flavour development in Cheddar cheese. Aust. J. Dairy Technol., 38, 57-59.

Aston J.W., Grieve P.A., Durward I.G., Dulley J.R., 1983b. Proteolysis and flavour development in Cheddar cheese subjected to accelerated ripening treatments. Aust. J. Dairy Technol., 38, 59-65.

Baraud J., Emassieux S., Maurice A., 1970. Biosynthèse des acides gras à chaîne impaire ou ramifiée chez la levure Schizosaccharomyces liquefaciens. Rev. Fr. Corps Gras, 17, 155-163.

Berdague J.L., Jeunet R., Grappin R., 1987. Affinage et qualité du Gruyère de Comté. III : Fermentation lactique et teneur en acides gras volatils des fromages de Comté. Lait, $67(2), 249-263$.

Снамва J.F., 1982. Le salage de l'Emmental. Etude de l'absorption, de la migration, de la répartition du sel et de son influence sur la qualité du fromage. Thèse de doctorat d'Université, Nancy I.

Collin J.C., Berdague J.L., Dognin-Bergeret M., Grappin R., 1987. Affinage et qualité du Gruyère de Comté, IV : Etude de la protéolyse. Lait, 67 (3),299-318.

DRIESSEN F.M., 1983. Lipases and proteinases in milk, occurrence, heat inactivation and their importance for the keeping quality of milk products. Thesis, September 1983. Verslag V236. Nederlands Institut voor Zwivelonderzoeck.

EGel W.N. 1977. Formation de $\gamma 1-\mathrm{A} 2, \gamma 2-\mathrm{A} 2$ et $\gamma 3-\mathrm{A} 2$ caseins by in vitro proteolysis of casein A2 with bovine plasmin. Int. J. Biochem., 8, 187-192.

FoX P.F., WALLEY B.F., 1971. Influence of sodium chloride on the proteolysis of casein by rennet and by pepsin. J. Dairy Res., 38, 165-170.

Grappin R., Rank T.C., Olson N.F., 1985. Primary proteolysis of cheese proteins during ripening. A review. J. Dairy Sci., 68, 531-540.

Grappin R., Berdague J.L., Dasen A., Jeunet R., 1987. Affinage et qualité du Gruyère de Comté. I ₹ Protocole expérimental. Caractérisation des conditions d'affinage et des ateliers de fabrication. Lait, 67 (2), 219-235.

Guichard E., Berdague J.L., Grappin R., 1987. Affinage et qualité du Gruyère de Comté. V : Influence de l'affinage sur la teneur en composés volatils. Lait, 67 (3), 319-338.

Kaminogawa S., Yamauchi K., 1972a. Decomposition of $\beta$-casein by milk protease. Similarity of the decomposed products to temperature sensitive and R-caseins. Agric. Biol. Chem., 36, 255260 .

Kaminogawa S., Yamauchi K., 1972b. Acid protease of bovine milk. Agric. Biol. Chem., 36, 2351-2356.

Kaminogawa S., Yamauchi K., Mirazawa S., Koya Y., 1980. Degradation of casein components by acid protease of bovine milk. J. Dairy Sci., 63, 701-704.

NoOMEn A., 1978. Activity of proteolytic enzymes in simulated soft cheeses (Meshanger type). 1: Activity of milk protease. Neth. Milk Dairy J., 32, 26-48.

Phelan J.A., Guiney J., Fox P.F., 1973. Proteolysis of $\beta$-casein in Cheddar cheese. J. Dairy Res., 40. $105-112$.

Stadhouders J., Hup G., 1975. Factors affecting bitter flavour in Gouda cheese. Neth. Milk Dairy J., 29, 335-353.

Stadhouders J., Hup G., Exterkate F.A., Visser S., 1983. Bitter flavour in cheese. 1. Mechanism of the formation of the bitter flavour defect in cheese. Neth. Milk Dairy J., 37, 157-167.

SUHREN G., 1983. Occurrence and levels of heat resistant proteinases and their effects on UHT treated dairy products. Int. Dairy Fed. Bull., F-Doc 85, 18-1.

Sullivan J.J., Jago G.R., 1972. The structure of bitter peptides and their formation from casein. Aust. J. Dairy Technol., 27, 98-103.

Visser S., Slangen K.J., Hup G., Stadhouders J., 1983. Bitter flavour in cheese. 3 : Comparative gel-chromatographic analysis of hydrophobic peptide fractions from twelve Gouda-type cheeses and identification of bitter peptides isolated from a cheese made with Streptococcus cremoris strain HP 1.2. Neth. Milk Dairy J., 37, 181-192. 